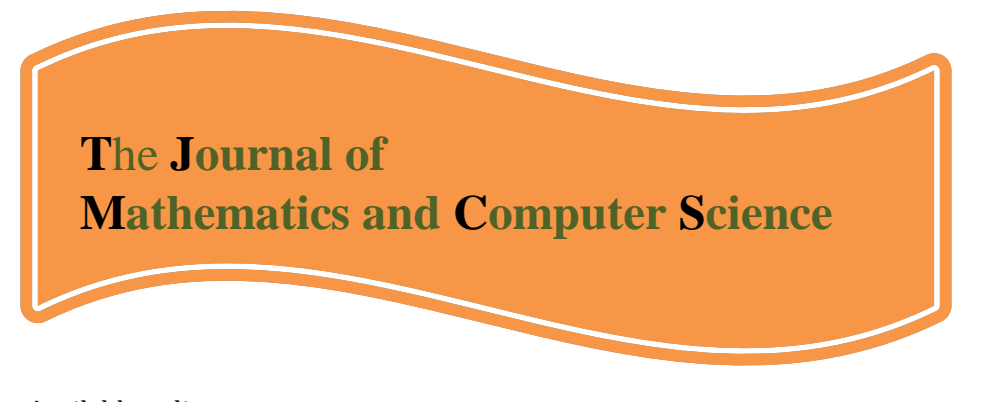

Available online at

\title{
http://www.TIMCS.com
}

\section{Solving a Class of Nonlinear Optimal Control Problems by Differential Transformation Method}

\author{
A. Fakharzadeh \\ Department of Mathematics, Shiraz University of Technology \\ a_fakharzadeh@sutech.ac.ir \\ S. Hashemi \\ Department of Mathematics, Shiraz University of Technology \\ s.hashemi@sutech.ac.ir
}

Received: February 2012, Revised: November 2012

Online Publication: December 2012

\begin{abstract}
Based on the Differential Transformation Method (DTM), a solution procedure for solving a class of nonlinear quadratic optimal control problems is presented in this paper. The reason for selecting this solution procedure is the less computational cost in comparison with the ordinary solution methods of original problem. First, the problem is converted to a two-point boundary value problem then the new problem is transferred into a set of algebraic equations by applying the differential transformation properties. By presenting the algorithmic solution procedure, two numerical examples are given to demonstrate the simplicity and efficiency of the new method.
\end{abstract}

Keywords: Quadratic optimal control problems; Pontryagin's maximum principle; Differential transformation method; recursive relations

2010 Mathematics Subject Classification: Primary 54A40; Secondary 46S40.

\section{Introduction}

Optimal control theory has been widely used in different fields such as biomedicine [1], aircraft systems [2], robotic [3] and etc. But in all these areas, the nonlinear systems are a challenging task which has been studied extensively for decades. In many cases, the optimal control law can be derived by using the famous Pontryagin's maximum principle. However, for the nonlinear OCP's, this approach leads to a nonlinear two-point boundary value problem (TPBVP), that unfortunately 
in general cannot be solved analytically. Therefore, many researchers have tried to find an approximate solution for these nonlinear TPBVP's. Regarding this fact, in the recent years, some new applications of finding an approximate analytical solution methods for TPBV differential systems have been presented to solve the related optimal control problems, such as Variational Iteration Method [4], Homotopy method [5], Optimal Homotopy Perturbation Method [6]. In sequential, here, we employ DTM to solve a class of nonlinear optimal control problems.

The organization of the paper is as follows. In section 2, we discussed the quadratic nonlinear OCP and its necessary and sufficient extreme conditions, and then the differential transformation method is explained in next section. Section 4 is devoted to present a DT-Based Algorithm for solving TPBVP. Section 5 presents two useful numerical examples in order to demonstrate the application of the method and its efficiency; finally section 6 concludes the paper.

\section{Problem statement}

A nonlinear quadratic optimal control problem in a general form can be presented as [7]:

$$
\begin{aligned}
& \text { Min: } J[x, u]=\frac{1}{2} \int_{t_{0}}^{t_{f}}\left(x^{T}(t) Q x(t)+u^{T}(t) R u(t)\right) d t, \\
& \text { S.to: } \quad \dot{x}=f(t, x(t))+g(t, x(t)) u(t),
\end{aligned}
$$

with $x(t) \in \mathbb{R}^{n}$ denoting the state variable, $u(t) \in \mathbb{R}^{m}$ denoting the control variable and $x_{0}, x_{f}$ are the initial and final states at $t_{0}, t_{f}$. Moreover $f$ and $g$ are two continuously differentiable functions in all arguments and $Q \in \mathbb{R}^{n \times n}, R \in \mathbb{R}^{m \times m}$ are positive semi-definite and positive definite matrices, respectively.

According to the Pontryagin's maximum principle [8], the optimality conditions for (1) are determined by the following nonlinear TPBVP:

$\dot{x}=f(t, x)+g(t, x)\left[-R^{-1} g^{T}(t, x) \lambda\right]$;

$\dot{\lambda}=-\left(Q x+\left(\frac{\partial f(t, x)}{\partial x}\right)^{T} \lambda+\sum_{i=1}^{n} \lambda_{i}\left[-R^{-1} g^{T}(t, x) \lambda\right]^{T} \frac{\partial g_{i}(t, x)}{\partial x}\right) ;$

$x\left(t_{0}\right)=x_{0} ; x\left(t_{f}\right)=x_{f}$,

where $\lambda(t) \in \mathbb{R}^{n}$ is the co-state vector with the $\mathrm{i}$-th component $\lambda_{i}(t), i=1,2, \ldots, n$ and $g(t, x)=\left[g_{1}(t, x), \ldots, g_{n}(t, x)\right]^{T}$ with $g_{i}(t, x) \in \mathbb{R}^{m}$. Also the optimal control law is demonstrated by $u^{*}=-R^{-1} g^{T}(t, x) \lambda$.

The system (2) contains a nonlinear TPBVP that in general cannot be solved analytically except in a few simple cases. In order to overcome this difficulty, we incline to apply the DTM approach to solve it. Therefore, the next section is devoted to illustrate this method.

\section{Differential Transformation Method}

We know that a (piecewise) smooth function $\mathrm{u}(\mathrm{x})$ can be expended in Taylor series about $\mathrm{x}=0$ as:

$u(x)=\sum_{k=0}^{\infty} \frac{x^{k}}{k !}\left(\frac{d^{k} u}{d x^{k}}\right)_{x=0}$.

Hence, we define the differential transformation of $\mathrm{u}(\mathrm{x})$ as:

$\mathrm{U}(k)=\frac{1}{k !}\left(\frac{d^{k} u}{d x^{k}}\right)_{x=0}$.

Then one can deduce that the inverse differential transform of $U(k)$ is: 
$u(x)=\sum_{k=0}^{\infty} x^{k} U(k)$.

Based on the above definitions, the fundamental mathematical operations performed by differential transform method is shown from [9] in the following Table.

Table 1. SOME DIFFERENTIAL TRANSFORMATION RELATIONSHIPS

\begin{tabular}{|c|c|}
\hline Original function & Transformed function \\
\hline$u(x)=w(x) \pm v(x)$ & $U(k)=W(k) \pm V(k)$ \\
\hline$u(x)=\alpha v(x)$ & $U(k)=\alpha V(k)$ \\
\hline$u(x)=\frac{d v(x)}{d x}$ & $U(k)=(k+1) V(k+1)$ \\
\hline$u(x)=x^{m}$ & $U(k)=\delta(k-m)= \begin{cases}1, & k=m \\
0, & k \neq m\end{cases}$ \\
\hline$u(x)=v_{1}(x) v_{2}(x) \ldots v_{n}(x)$ & $U(k)=\sum_{k_{n-1}=0}^{k} \sum_{k_{n-2}=0}^{k_{n-1}} \ldots \sum_{k_{2}=0}^{k_{3}} \sum_{k_{1}=0}^{k_{2}} V_{1}\left(k_{1}\right) V_{2}\left(k_{2}-k_{1}\right) \ldots V_{n-1}\left(k_{n-1}-k_{n-2}\right) V_{n}\left(k-k_{n-1}\right)$ \\
\hline$u(x)=\sin (c x+\beta)$ & $U(k)=\frac{c^{k}}{k !} \sin \left(\frac{\pi k}{2}+\beta\right)$ \\
\hline$u(x)=\cos (c x+\beta)$ & $U(k)=\frac{c^{k}}{k !} \cos \left(\frac{\pi k}{2}+\beta\right)$ \\
\hline
\end{tabular}

\section{DT-Based Algorithm for solving the TPBVP}

To solve (2) by DT approach, first we solve the following initial value problem, using DT method,

$\dot{x}=f(t, x)+g(t, x)\left[-R^{-1} g^{T}(t, x) \lambda(t)\right] ;$

$\dot{\lambda}=-\left(Q x+\left(\frac{\partial f(t, x)}{\partial x}\right)^{T} \lambda+\sum_{i=1}^{n} \lambda_{i}\left[-R^{-1} g^{T}(t, x) \lambda\right]^{T} \frac{\partial g_{i}(t, x)}{\partial x}\right) ;$

$x\left(t_{0}\right)=x_{0} ; \lambda\left(t_{0}\right)=\alpha$,

where $\alpha \in \mathbb{R}^{n}$ is an unknown parameter to be determined. For the sake of simplicity, let us define the right hand sides of (7) as follows:

$w(t, x, \lambda)=f(t, x)+g(t, x)\left[-R^{-1} g^{T}(t, x) \lambda(t)\right] ;$

$h(t, x, \lambda)=-\left(Q x+\left(\frac{\partial f(t, x)}{\partial x}\right)^{T} \lambda+\sum_{i=1}^{n} \lambda_{i}\left[-R^{-1} g^{T}(t, x) \lambda\right]^{T} \frac{\partial g_{i}(t, x)}{\partial x}\right) ;$

$x\left(t_{0}\right)=x_{0} ; \lambda\left(t_{0}\right)=\alpha$,

Thus the (7) changes to:

$\dot{x}=w(t, x, \lambda)$;

$\dot{\lambda}=h(t, x, \lambda)$;

$x\left(t_{0}\right)=x_{0} ; \lambda\left(t_{0}\right)=\alpha$,

According to DTM, for solving an initial value problem such as (9) we can base on the rules mentioned in Table1 construct a recursive relation as follows: 


$$
\begin{aligned}
& (k+1) X(k+1)=W(k) ; \\
& (k+1) \Lambda(k+1)=\mathrm{H}(k) ; \\
& X(0)=x_{0}, \Lambda(0)=\alpha .
\end{aligned}
$$

Which in that $X, \Lambda, W$ and $H$ are the differential transfer of $x, \lambda, w$ and $h$ respectively.

We present the following algorithm for solving TPBVP's:

Step1. Let $X(0)=x\left(t_{0}\right), \Lambda(0)=\alpha$ and determine $\mathrm{N}$, ( $\mathrm{N}$ is the number of terms to be kept in the Taylor series.)

Step2. Obtain the differential transformation form (9) based on the rules mentioned in Table1. Determine the recursive relations for $X(k), \Lambda(k)$.

Step3. Calculate $X(k)$ and $\Lambda(k)$ for $k=1,2, . ., N$ from the obtain recursive equations in step 2 .

Step4. Construct $x(t)=\sum_{k=0}^{N} \frac{X(k)}{k !} t^{k}$ and $\lambda(t)=\sum_{k=0}^{N} \frac{\Lambda(k)}{k !} t^{k}$.

Step5: Solve the equation $x\left(t_{f}\right)=x_{f}$ to compute an approximation value for $\alpha$.

If $x\left(t_{f}\right)$ is free, solve $\lambda\left(t_{f}\right)=0$ to calculate an approximation value for $\alpha$.

Step6. Determine the optimal control solution by the optimal control law $u^{*}(t)=-R^{-1} g^{T} \lambda(t)$.

\section{Numerical examples and comparison}

To show the advantages of the mentioned new method in the previous section, here we present numerical simulations of two different cases. The first ones, has the terminal condition and in the other one, the terminal condition is free. The selected examples were solved in [4] by VIM. More than our new method, we solved the related TPBVP's by fourth-order Runge-Kutta method as well to have a suitable comparison. We applied MAPLE 13 for calculating the coefficient of the recursive equations.

Example 5.1. Consider the following quadratic optimal control problem with the give initial and terminal conditions:

Min : $J=\int_{0}^{1} u^{2}(t) d t$

S.to : $\dot{x}=\frac{1}{2} x^{2}(t) \sin x(t)+u(t), t \in[0,1]$;

$$
x(0)=0, x(1)=0.5 \text {. }
$$

By apply the PMP, we could reach to the following co-state system:

$$
\begin{aligned}
& \dot{x}(t)=\frac{1}{2} x^{2}(t) \sin x(t)-\frac{1}{2} \lambda(t), t \in[0,1] ; \\
& \dot{\lambda}(t)=-\lambda(t) x(t) \sin x(t)-\frac{1}{2} \lambda(t) x^{2}(t) \cos x(t) ; \\
& x(0)=0, \lambda(0)=\alpha .
\end{aligned}
$$

By regarding (3) the optimal control law for this problem is $u^{*}(t)=-\frac{1}{2} \lambda(t)$. We know that here $\alpha$ is an unknown parameter to be determined. According to Table 1, the following recursive formulas are determined: 


$$
\begin{aligned}
& X(k+1)=\frac{1}{2(k+1)}\left[\sum_{k_{2}=0}^{k} \sum_{k_{1}=0}^{k_{2}} \frac{1}{k_{1} !} \sin \left(\frac{\pi k_{1}}{2}\right) X\left(k_{2}-k_{1}\right) X\left(k-k_{2}\right)-\Lambda(k)\right] ; \\
& \Lambda(k+1)=\frac{1}{(k+1)}\left[-\sum_{k_{2}=0}^{k} \sum_{k_{1}=0}^{k_{2}} \frac{1}{k_{1} !} \sin \left(\frac{\pi k_{1}}{2}\right) \Lambda\left(k_{2}-k_{1}\right) X\left(k-k_{2}\right)\right. \\
& \left.-\frac{1}{2} \sum_{k_{3}=0}^{k} \sum_{k_{2}=0}^{k_{3}} \sum_{k_{1}=0}^{k_{2}} \frac{1}{k_{1} !} \cos \left(\frac{\pi k_{1}}{2}\right) X\left(k_{2}-k_{1}\right) X\left(k_{3}-k_{2}\right) \Lambda\left(k-k_{3}\right)\right] ; \\
& X(0)=0, \quad \Lambda(0)=\alpha .
\end{aligned}
$$

By means of DTM algorithm with $N=3, \alpha=-1$ was obtained. Simulated curves of the state trajectory and control law are computed and shown in figure 1 and figure 2, respectively. Moreover they compared with simulation curves that computed by fourth-order Runge-Kutta method.

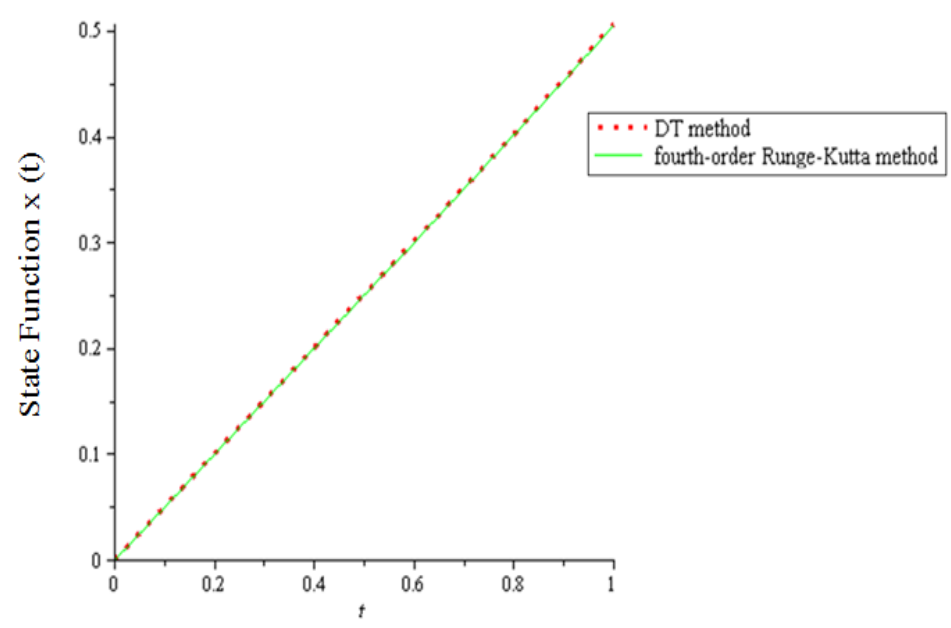

Figure 1.The suboptimal state $x(t)$, Example 5.1.

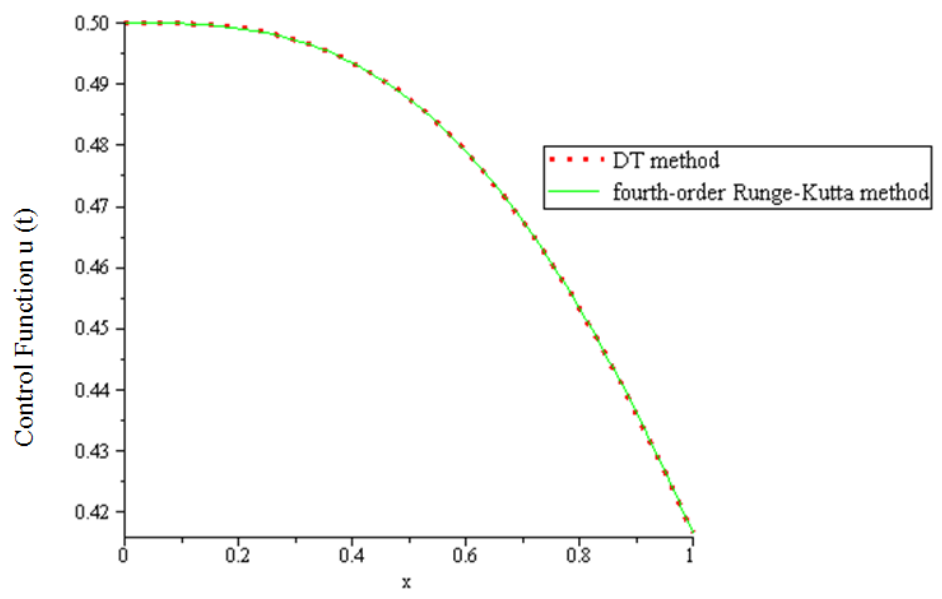

Figure 2.The suboptimal control u(t), Example 5.1.

In comparison with [4], the curves are similar but the computations and consumed time is less.

Example 5.2. We considered the nonlinear quadratic optimal control problem where its terminal time is free, described as follows: 
$\operatorname{Min} J=\frac{1}{2} \int_{0}^{1}\left(x_{1}^{2}+x_{2}^{2}+u^{2}\right) d t$,

$$
\begin{aligned}
& \dot{x}_{1}=x_{2}+x_{1} x_{2} ; \\
& \dot{x}_{2}=-x_{1}+x_{2}+x_{2}^{2}+u ; \\
& x_{1}(0)=-0.8, x_{2}(0)=0 .
\end{aligned}
$$

By apply the PMP, we could reach to the following co-state system:

$\dot{\lambda}_{1}=-\left(x_{1}+\lambda_{1} x_{2}-\lambda_{2}\right)$;

$\dot{\lambda}_{2}=-\left(x_{2}+\lambda_{1}\left(1+x_{1}\right)+\lambda_{2}\left(1+2 x_{2}\right)\right)$;

$\dot{x}_{1}=x_{2}+x_{1} x_{2}$

$\dot{x}_{2}=-x_{1}+x_{2}+x_{2}^{2}-\lambda_{2}$;

$x_{1}(0)=-0.8, x_{2}(0)=0$,

$\lambda_{1}(1)=\lambda_{2}(1)=0$.

Here the optimal control is $u^{*}(t)=-\lambda(t)$. With help of DTM algorithm, which described in section 4 , the obtained optimal trajectories and control are shown in figures 3, 4 and 5 respectively. They also compared with simulation curves that computed by fourth-order Runge-Kutta method.

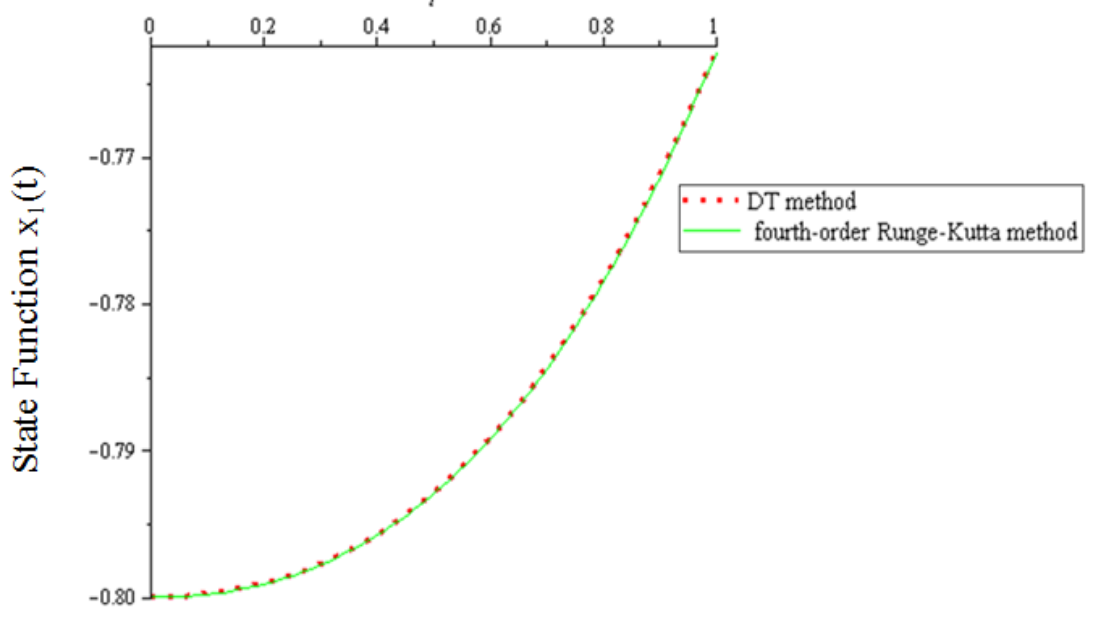

Figure 3.The suboptimal state $x_{1}(t)$, Example 5.2.

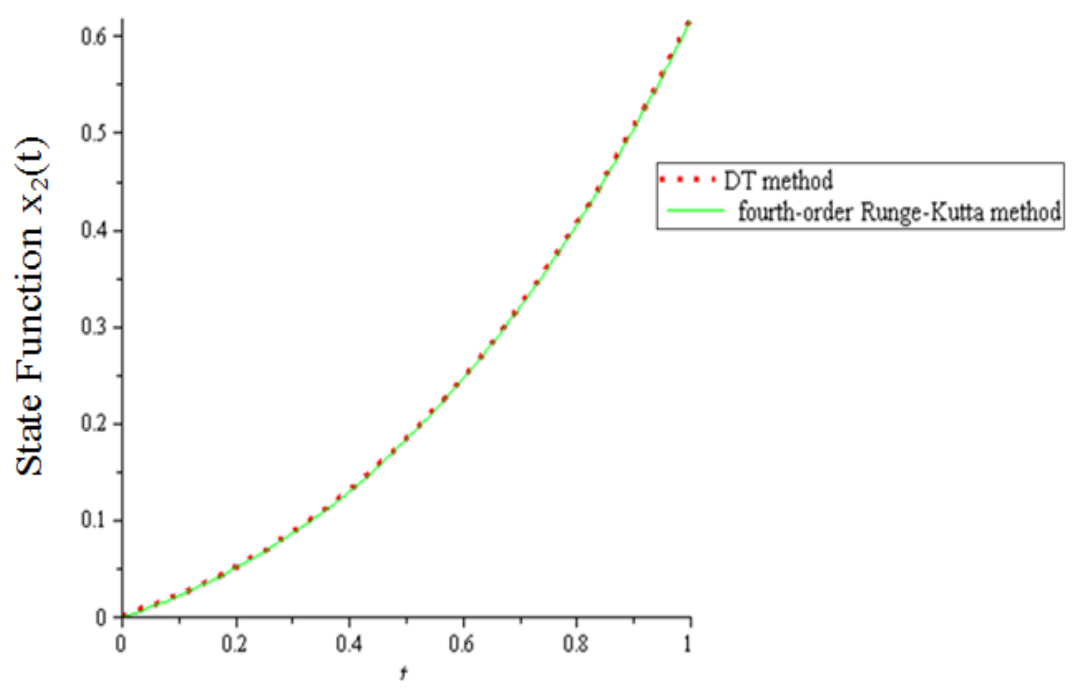

Figure 4.The suboptimal state $x_{2}(t)$, Example 5.2. 


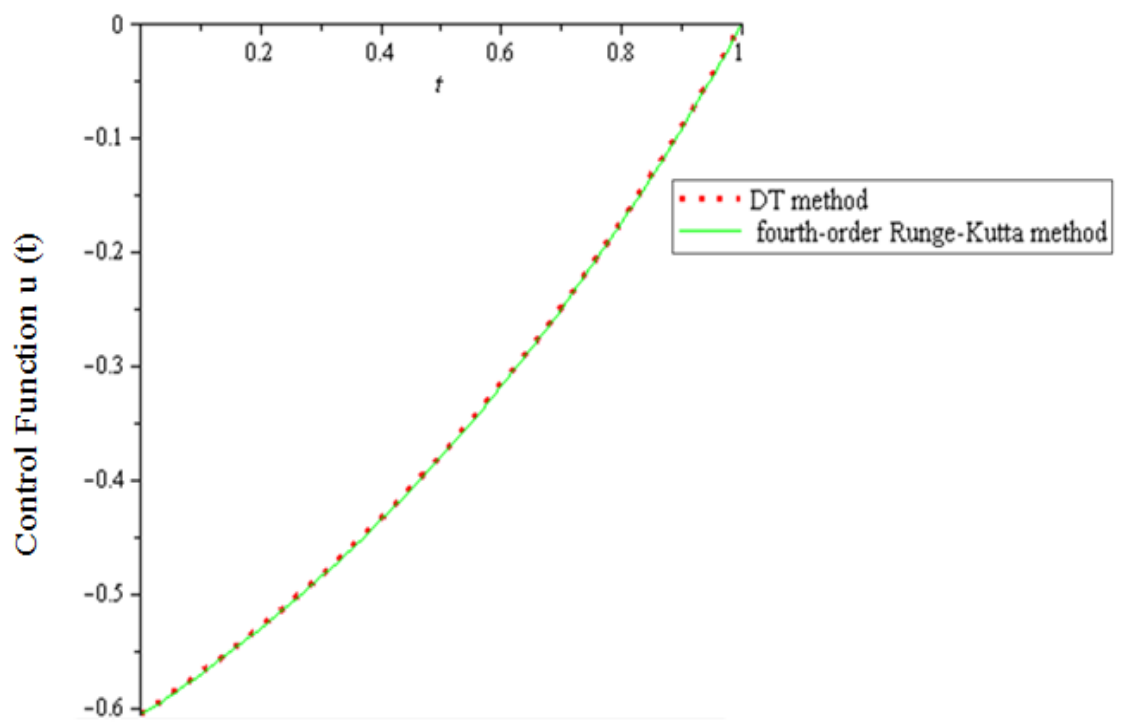

Figure 5.The suboptimal control u(t), Example 5.2

\section{Conclusions}

We applied the DT approaches to solve an important class of nonlinear quadratic optimal control problems. Compared with the presented classical solution method for such problems, the DTM has much less computation with remarkable simplicity. This method is an approximated analytical solution as a differential series in which the coefficients can be computed much easy and accurate. Also, the comparison results with the simulations with VIM and direct use of fourth-order RungeKutta method, conformed these facts.

\section{References.}

[1] M. Itik, M. U. Salamci and S. P. Banks, Optimal control of drug therapy in cancer treatment, Nonlinear Analysis, Vol. 71, No. 12, pp. 1473-1486, 2009.

[2] W. L. Garrard, and J. M. Jordan, Design of nonlinear automatic flight control systems, Automatica, Vol. 13, No. 5, pp. 497-505, 1977.

[3] S. Wei, M. Zefran and R. A. DeCarlo, Optimal control of robotic system with logical constraints: application to UAV path planning, In Proceeding(s) of the IEEE International Conference on Robotic and Automation, Pasadena, CA, USA, pp.249-256, 2008.

[4] M. Shirazian, and S. Effati, Solving a Class of Nonlinear Optimal Control Problems via He's Variational Iteration Method, International Journal of Control, Automation, and Systems,2012.

[5] S. Effati and H. Saberi Nic, Solving a class of linear and non-linear optimal control problems by homotopy, IMA Journal of Mathematical Control and Information, Vol.28, No.2, pp.539-553, 2011.

[6] A. Jajarmi, N. Pariz and A. VahidianKamyad, A highly computational efficient method to solve nonlinear optimal control problems, ScientiaIranica, 2011.

[7] D. E. Kirk, Optimal Control Theory, An Introduction, New Jersi, 1970.

[8] E. R. Pinch, Optimal Control and the Calculus of Variations, Oxford University Press, 1993.

[9] R. Abazari and R. Abazari, Numerical study of some coupled PDEs by usingdifferential transformation method, Engineering and Technology 66Management, 2010. 